\title{
Perfil sensorial e aceitação de bebida láctea achocolatada
}

\author{
Sensory profile and acceptance of milk chocolate beverage
}

\section{Sérgio Bertelli PFLANZER ${ }^{1 *}$, Adriano Gomes da CRUZ ${ }^{1}$, Camila Lie HATANAKA ${ }^{1}$, Priscila Lourezon MAMEDE Rafael CADENA ${ }^{1}$, José Assis Fonseca FARIA ${ }^{1}$, Maria Aparecida Azevedo Pereira da SILVA ${ }^{1}$}

\section{Resumo}

O perfil sensorial de bebida láctea achocolatada foi obtido por meio da Análise Descritiva Quantitativa (ADQ), utilizando três marcas comerciais do produto. Doze termos descritivos foram desenvolvidos por uma equipe de provadores selecionados e treinados, que geraram também a definição de cada termo e as amostras referência. Os resultados foram submetidos à ANOVA, teste de Tukey e Análise de Componentes Principais. A bebida láctea achocolatada da marca A caracterizou-se por apresentar maior intensidade de doçura, aroma e sabor de caramelo, enquanto as características predominantes da marca B foram a cor marrom, viscosidade e arenosidade muito intensos. O produto da marca C diferiu dos demais por apresentar maior aroma e sabor de leite. Foi também realizado um teste de aceitação para avaliar as amostras de uma forma global, quanto a intensidade do ideal de doçura e a intenção de compra. Todas as marcas apresentaram boa aceitabilidade global, não apresentando diferença significativa entre si, assim como a intenção de compra. O teste com o consumidor indicou que a amostra B apresentou intensidade de doçura ideal.

Palavras-chave: bebida láctea achocolatada; análise sensorial; análise descritiva quantitativa; aceitação; doçura.

\begin{abstract}
The sensory profile of three commercial brands of milk chocolate beverage was obtained using the Quantitative Descriptive Analysis (QDA). Twelve descriptive terms, their definitions, and reference samples were generated by a selected and trained panel. The statistical analysis included the Analysis of Variance (ANOVA), Tukey's Test for means, and Principal Component Analysis (PCA). Brand A showed strong sweetness taste, aroma, and caramel flavor, whereas the predominant characteristics of brand B were the brown color, viscosity, and very intense sandiness. Brand $\mathrm{C}$ differed from the others for presenting stronger aroma and milk flavor. An acceptance test was also performed to evaluate the samples thoroughly in terms of the intensity of the ideal sweetness and buying intention. All brands presented high acceptability, and there was no significant difference between them.

Keywords: chocolate milk beverage; sensory analysis; descriptive quantitative analysis; acceptance; sweetness.
\end{abstract}

\section{Introdução}

A bebida láctea é o produto resultante da mistura de leite (in natura, pasteurizado, esterilizado, UHT, reconstituído, concentrado, em pó, integral, semidesnatado, ou parcialmente desnatado) e soro de leite (líquido, concentrado ou em pó) acrescido ou não de produtos, substâncias alimentícias, gordura vegetal, leite fermentado, fermentos lácteos selecionados e outros produtos lácteos (BRASIL, 2005). Constitui-se em uma forma racional e lógica de aproveitamento do soro de queijo para retorno à cadeia humana de forma palatável, sem prejuízo ao meio ambiente (ALMEIDA; BONASSI; ROÇA, 2001).

As bebidas contendo soro de queijo são, hoje, uma realidade no mercado brasileiro, sendo processadas de diversas maneiras como UHT, pasteurizadas, fermentadas semelhantes ao iogurte, soft-drinks, carbonatadas e em diversos sabores, como morango, chocolate, frutas cítricas, etc., com um mercado consumidor bastante promissor (LIMA; MADUREIRA; PENNA, 2002). Desta forma, é crescente o número de pesquisas relatando o desenvolvimento e estabilidade de bebidas contendo soro de queijo (YANES; DURÁN; COSTELL, 2002; SIVIERI; OLIVEIRA, 2002; ERNEST et al., 2005; PIFARRE et al., 2006; CASTRO et al., TRANJAN et al., 2009; CRUZ et al., 2009; ZOELNNER et al., 2009; KOFFI; SHEWFELT; WICKER, 2005).

Um levantamento recente indica que bebidas lácteas fermentadas já representam $25 \%$ do mercado total de iogurtes no Brasil. Quando lançadas tinham como objetivo atrair consumidores da Classe C e D. Com o Plano Real, o atrativo "preço" foi uma das principais razões do crescimento do mercado deste produto, o que possibilitou, inclusive, o consumo dos produtos pela Classe E. Em particular, o mercado de bebidas lácteas achocolatadas movimentou um volume de 52 milhões de L em 1995 e tem experimentado um crescimento significativo de 85,5\% nos últimos anos (RITJENS, 1997).

A análise sensorial é um método científico utilizado para medir, analisar e interpretar as características dos alimentos que

${ }^{1}$ Faculdade de Engenharia de Alimentos, Universidade Estadual de Campinas - UNICAMP, CEP 13083-862 Campinas - SP, Brasil, E-mail: spflanzer@gmail.com

${ }^{*}$ A quem a correspondência deve ser enviada 
possam ser percebidas pelo sentido do paladar, visão, olfato, tato e audição, utilizando conhecimentos de Ciência de Alimentos, Fisiologia, Psicologia e Estatística (SIMPSON; PIGGOTT; WILLIAMS, 1998). Pode ser dividida em duas áreas: objetiva (analítica) e subjetiva (hedônica). No primeiro, os atributos sensoriais do produto são avaliados e selecionados por um painel de provadores treinados. No segundo, são avaliadas as reações dos consumidores aos produtos (KEMP, 2008).

Nos últimos anos, a análise sensorial deixou de ser uma atividade secundária e empírica e enquadrou-se na categoria de disciplina científica, capaz de gerar informações precisas e reprodutíveis. Sobre estas informações recaem importantes decisões como seleção da matéria-prima, padronização de métodos e otimização de formulações, para desenvolvimento de produtos, tornando-se assim uma ferramenta básica para aplicação na indústria de alimentos (MEHINAGIC et al., 2003).

O objetivo deste trabalho foi determinar o perfil sensorial por meio da Análise Descritiva Quantitativa (ADQ) e a aceitação de bebidas lácteas achocolatadas.

\section{Material e métodos}

\subsection{Amostras}

Foram avaliadas três marcas comerciais de bebida láctea achocolatada, envasadas em embalagem UHT de $1 \mathrm{~L}$, adquiridas em supermercados da região de Campinas - SP. Os testes sensoriais foram realizados no Laboratório de Análise Sensorial da Faculdade de Engenharia de Alimentos (FEA/UNICAMP), cujas instalações incluem cabinas individuais e controle de iluminação e temperatura ambiente.

O critério de escolha das bebidas achocolatadas foi seu preço e seu conhecimento pelo consumidor. Desta forma, foram escolhidos produtos que atendessem a esse objetivo. Foram escolhidas três amostras: uma amostra considerada líder de mercado (B), com preço aproximado de $\mathrm{R} \$ 4,50$; as outras duas amostras selecionadas têm preços semelhantes entre si (R \$2,70), sendo que uma delas (C) possui uma melhor qualidade reconhecida pelos consumidores e está há mais tempo no mercado, e a amostra A caracteriza-se por ser uma nova concorrente no mercado.

O perfil sensorial de cada amostra de bebida láctea achocolatada foi determinado utilizando a Análise Descritiva Quantitativa (ADQ) descrita por Stone e Sidel (1985), com provadores treinados. Foi ainda realizado teste de aceitação global e de sabor, intenção de compra e escala do ideal de doçura com a participação de consumidores do produto, conforme recomendações de Meilgaard, Civille e Carr (1987).

\subsection{Análise descritiva quantitativa}

\section{Recrutamento e pré-seleção dos provadores}

Os provadores foram recrutados entre os alunos que cursavam a disciplina de Análise Sensorial ministrada no curso de Pós-Graduação do Departamento de Alimentos e Nutrição da FEA/UNICAMP.

\section{Desenvolvimento da terminologia descritiva e treinamento dos provadores}

Para o levantamento inicial de termos que descrevessem a qualidade da bebida láctea achocolatada, foram utilizadas as 3 amostras a serem analisadas. Utilizando o método de Rede, segundo Moskowitz (1983), as 3 amostras foram apresentadas simultaneamente, solicitando-se ao provador que agrupasse as amostras aos pares e descrevesse as similaridades e as diferenças entre elas. Foi utilizada uma mesma ficha para aparência, aroma, sabor e textura (Figura 1).

Após cada provador ter gerado seus próprios termos, foi realizada uma discussão em grupo, sob a supervisão de um líder, com o objetivo de agrupar termos semelhantes e eliminar aqueles que não eram percebidos pela maioria dos provadores. Durante 3 sessões posteriores de avaliação das amostras, foram definidos os termos descritivos e as referências para melhor uniformizar a avaliação dos provadores (Tabela 1) e, também, a elaboração da Ficha de Avaliação (Figura 2) contendo os termos descritivos escolhidos em consenso pela equipe sensorial. Foi utilizada uma escala linear não estruturada de $9 \mathrm{~cm}$, ancorada nas suas extremidades com termos que expressam intensidade. Quando o descritor tinha a possibilidade de não estar presente em alguma das amostras, o extremo inferior da escala era chamado "nenhum".

\section{Seleção final da equipe de $A D Q$}

Nesta etapa foram avaliadas as três amostras de bebida láctea achocolatada, em delineamento experimental em blocos completos casualizados com três repetições. Os provadores utilizaram a própria Ficha de Avaliação desenvolvida na etapa

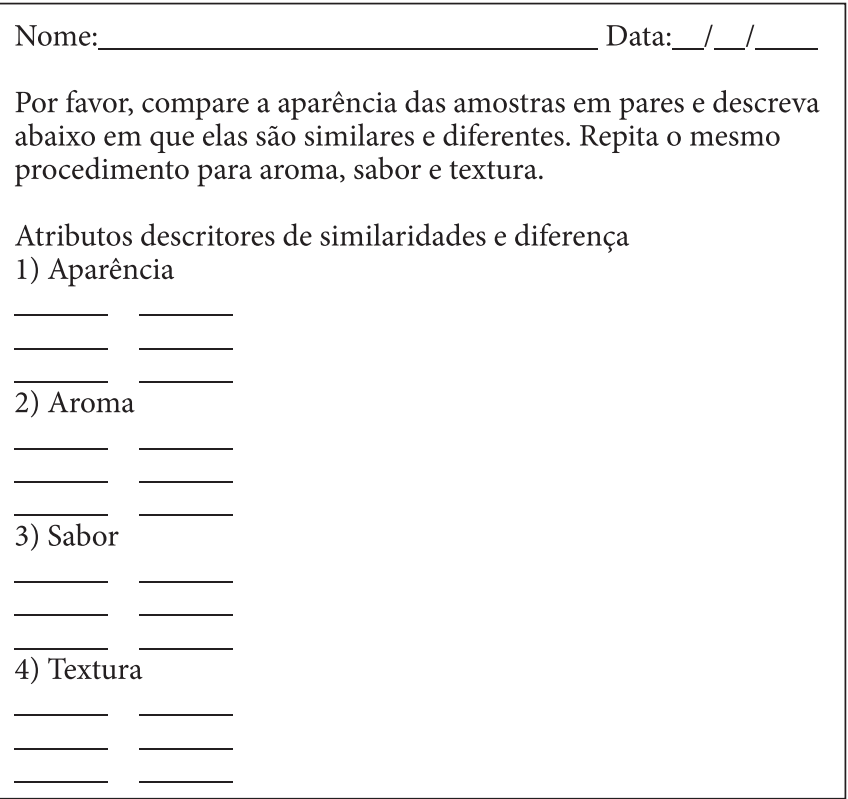

Figura 1. Ficha utilizada para o método Rede no levantamento dos termos descritivos. 
Tabela 1. Lista dos termos descritivos e respectivas referências.

\begin{tabular}{|c|c|c|}
\hline Atributo & Definição & Referências \\
\hline \multicolumn{3}{|l|}{ Aparência } \\
\hline Marrom & Intensidade da cor marrom & $\begin{array}{l}\text { Fraco: Achocolatado } \mathrm{SHEFA}^{\oplus}+30 \% \text { de leite UHT integral SHEFA. } \\
\text { Forte: Achocolatado TODDY }+7 \% \text { de chocolate em pó NESTLE }{ }^{\oplus} \text {. }\end{array}$ \\
\hline Brilho & $\begin{array}{l}\text { Capacidade de reflexão da luz na superfície do } \\
\text { produto }\end{array}$ & $\begin{array}{l}\text { Pouco: Barra de chocolate ao leite NESTLÉ } \\
\text { Muito:Barra de chocolate ao leite NESTLÉ } \\
\text { besuntada com óleo de soja. }\end{array}$ \\
\hline Viscosidade & Tempo de escoamento na borda do corpo & $\begin{array}{l}\text { Pouco: Leite UHT integral SHEFA }{ }^{\bullet} \text {. } \\
\text { Muito: Leite UHT integral SHEFA }+4 \% \text { de amido de milho MAIZENA }{ }^{\circ} \text {. }\end{array}$ \\
\hline \multicolumn{3}{|l|}{ Aroma } \\
\hline Chocolate & Aroma associado ao chocolate em pó & $\begin{array}{l}\text { Fraco: Solução de chocolate em pó NESTLÉ a } 0,25 \% \text {. } \\
\text { Forte: Solução de chocolate em pó NESTLÉ® a } 5 \% \text {. }\end{array}$ \\
\hline Leite & Intensidade do aroma característico de leite & $\begin{array}{l}\text { Fraco: Leite UHT integral SHEFA }{ }^{\oplus}+40 \% \text { de água. } \\
\text { Forte: Leite UHT integral SHEFA }{ }^{\oplus} \text {. }\end{array}$ \\
\hline Caramelo & Aroma associado à bala Tofee de caramelo & $\begin{array}{l}\text { Nenhum. } \\
\text { Muito: Bala Tofee. }\end{array}$ \\
\hline \multicolumn{3}{|l|}{ Sabor } \\
\hline Chocolate & Sabor associado ao chocolate em pó solúvel & $\begin{array}{l}\text { Fraco: Achocolatado SHEFA }{ }^{\oplus}+30 \% \text { de leite UHT Integral SHEFA } \\
\text { Forte: Achocolatado TODDY } \\
\text { Fo }+2 \% \text { de chocolate em pó NESTLÉ. }\end{array}$ \\
\hline Leite & Intensidade do sabor característico de leite & $\begin{array}{l}\text { Fraco: Leite UHT integral SHEFA }{ }^{\oplus}+40 \% \text { de água. } \\
\text { Forte: Leite UHT integral SHEFA }{ }^{\oplus} \text {. }\end{array}$ \\
\hline Caramelo & Aroma associado à bala Tofee de caramelo & $\begin{array}{l}\text { Nenhum. } \\
\text { Muito: Bala Tofee. }\end{array}$ \\
\hline Doçura & $\begin{array}{l}\text { Sensação do gosto básico provocado pela } \\
\text { sacarose }\end{array}$ & $\begin{array}{l}\text { Fraco: Achocolatado SHEFA }{ }^{\oplus}+50 \% \text { de leite UHT integral SHEFA }{ }^{\oplus} \text {. } \\
\text { Forte: Achocolatado TODDY }+2 \% \text { de açucar. }\end{array}$ \\
\hline \multicolumn{3}{|r|}{ - } \\
\hline Corpo & $\begin{array}{l}\text { Intensidade da sensação de preenchimento na } \\
\text { boca }\end{array}$ & $\begin{array}{l}\text { Fraco: Achocolatado } \mathrm{SHEFA}^{\oplus}+10 \% \text { de água. } \\
\text { Forte: Achocolatado TODDY }+3 \% \text { de amido de milho. }\end{array}$ \\
\hline Arenosidade & Presença de partículas perceptíveis em solução & $\begin{array}{l}\text { Nenhum: Leite UHT integral SHEFA }{ }^{\oplus} \text {. } \\
\text { Muito: Leite UHT integral SHEFA }+1,5 \% \text { de amido de milho MAIZENA }\end{array}$ \\
\hline
\end{tabular}

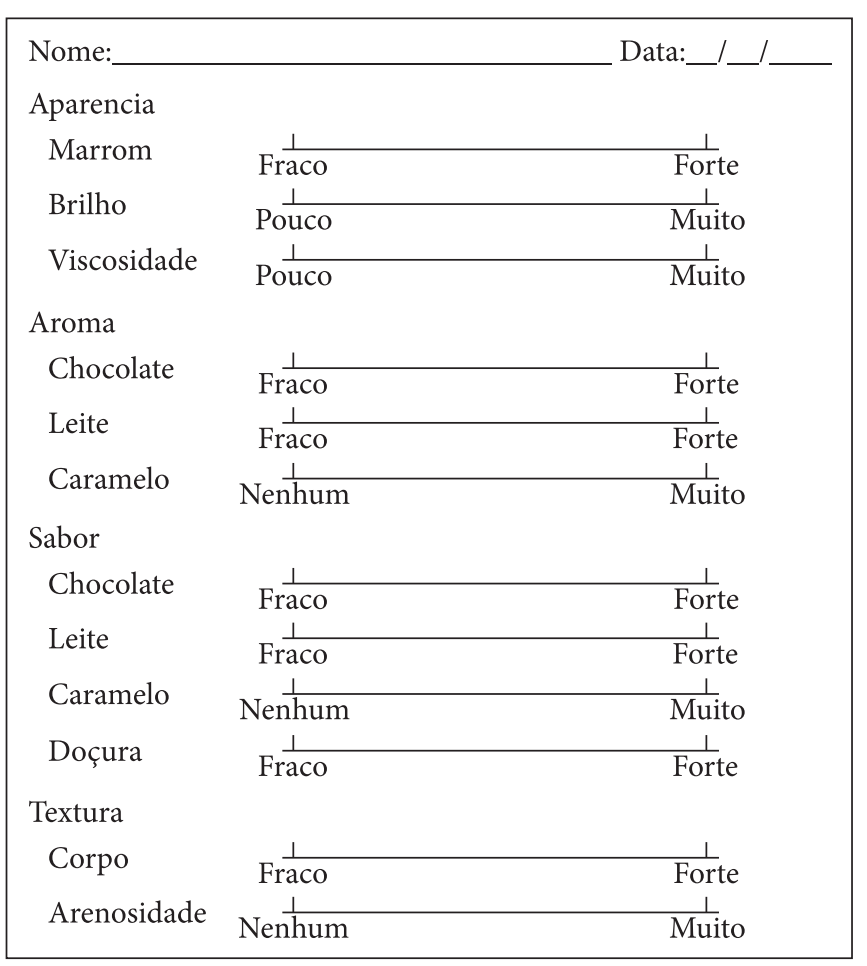

Figura 2. Ficha utilizada no treinamento e avaliação das amostras de bebida láctea achocolatada. anterior, sendo-lhes permitido consultar, a qualquer momento da sua análise, a lista de definições e as referências.

Foi então realizada a Análise de Variância para os resultados de cada provador, para cada descritor avaliado, tendo como fontes de variação amostras e repetições. Foram selecionados, para compor a equipe descritiva final, aqueles que apresentaram bom poder discriminativo $\left(\mathrm{P}_{\text {amostra }}<0,30\right)$; consenso com os demais membros do grupo e boa reprodutibilidade nos julgamentos $\left(\mathrm{P}_{\text {repetição }}>0,05\right)$, segundo metodologia proposta por Damasio e Costell (1991).

\section{Avaliação das amostras}

O experimento constou da avaliação sensorial de três amostras de bebida láctea achocolatada, por uma equipe de 5 provadores treinados. Foi utilizado delineamento em blocos completos casualizados com 3 repetições. Utilizou-se técnica de apresentação em blocos completos, ou seja, as três amostras foram apresentadas simultaneamente. A ordem de apresentação das amostras foi balanceada de forma a evitar vícios nos resultados.

Os provadores receberam amostras de aproximadamente $30 \mathrm{~mL}$ de achocolatado a $20{ }^{\circ} \mathrm{C}$, servidas em copinhos descartáveis codificados com números de três dígitos e um copo com água para eliminar resíduos do produto na boca. 


\subsection{Teste de aceitação}

A aceitabilidade das três amostras de bebida láctea achocolatada foi avaliada com a participação de 32 consumidores do produto, recrutados através da sua disponibilidade, interesse e frequência de consumo de produtos lácteos achocolatados. Antes da realização do teste, os consumidores eram advertidos sobre a presença de possíveis reações alérgicas devido à ingestão do produto, sendo possível a sua desistência em participar da análise. Adicionalmente, todos os consumidores que concordaram em participar do teste assinaram um termo de consentimento.

O teste foi realizado em laboratório, sob condições controladas. Cada provador recebeu aproximadamente $30 \mathrm{~mL}$ de bebida láctea achocolatada refrigerada, de forma monádica, sendo servida em copinho descartável codificado com números de três dígitos aleatórios, sendo-lhe solicitado provar o produto e avaliar a amostra de uma forma global (modo geral), e quanto ao sabor, utilizando uma escala hedônica estruturada mista de 9 pontos. Em seguida, foi solicitada a avaliação do produto quanto à intensidade de doçura, usando uma escala do ideal, $\mathrm{e}$ por último foi avaliada a intenção de compra, com uma escala de 5 pontos, caso o produto fosse encontrado à venda (Figura 3 ).

\subsection{Análises estatísticas}

Os resultados da $\mathrm{ADQ}$ foram submetidos à Análise de Variância (ANOVA), Teste de Tukey ao nível de 5\% de significância para a comparação entre as médias, a Análise de Componentes Principais. Os resultados do teste de aceitação foram analisados por meio de Análise de Variância e teste de Tukey para médias. Todas as análises estatísticas foram realizadas utilizando-se o programa estatístico SAS (1989).

\section{Resultados e discussão}

\subsection{Seleção de provadores e termos descritivos}

O desempenho dos provadores pode ser analisado através dos valores de $\mathrm{P}_{\text {amostra }}$ e $\mathrm{P}_{\text {repetição }}$ que são apresentados na Tabela 2. Observa-se que, de um modo geral, o desempenho dos provadores foi satisfatório. O provador 5 , apesar da ótima repetibilidade, apresentou baixo poder discriminatório e falta de consenso em 5 dos 12 atributos analisados, sendo, portanto eliminado. Os descritores aroma e sabor de baunilha provocaram, mesmo após treinamento, certa confusão entre os provadores, que não conseguiram chegar a um consenso em suas análises. Decidiu-se, então, eliminar estes descritores.

$A$ adoção da $A D Q$ requer um painel de provadores com um nível de treinamento e orientação, além de motivação e comprometimento. Estes devem, obrigatoriamente, frequentar as sessões de avaliação dos produtos, mesmo que tenham bom desempenho nessas sessões (MURRAY; DELAHUNTY; BAXTER, 2001).

\subsection{Perfil sensorial da bebida láctea achocolatada}

Os resultados da Análise de Variância das notas atribuídas pelos provadores a cada descritor, para cada uma das marcas

\begin{tabular}{|c|c|}
\hline \multicolumn{2}{|l|}{ Nome:- } \\
\hline \multirow{2}{*}{\multicolumn{2}{|c|}{$\begin{array}{l}1 \text { - Você está recebendo uma amostra de bebida láctea achocolatada, prove } \\
\text { a amostra através da escala acima expesse quanto você gostou ou desgostou } \\
\text { da amostra de um modo geral } \\
\quad \text { Valor: } \\
2 \text { - Descreva o que você mais gostou e menos gostou na amostra de um } \\
\text { modo geral } \\
\text { Mais gostou: } \\
\text { Menos gostou: }\end{array}$}} \\
\hline & \\
\hline \multicolumn{2}{|c|}{$\begin{array}{l}3 \text { - Porve a amostra novamente através da escala acima aroma expresse } \\
\text { quanto você gostou ou desgostou na amostra quanto ao sabor } \\
\text { Valor: }\end{array}$} \\
\hline \multicolumn{2}{|c|}{$\begin{array}{l}4 \text { - Descreva o que você mais gostou e menos gostou na amostra quanto ao } \\
\text { sabor } \\
\text { Mais gostou: }\end{array}$} \\
\hline \multicolumn{2}{|l|}{ Menos gostou: } \\
\hline $\begin{array}{l}5 \text { - Prove a amostra novamente e } \\
\text { através da escala ao lado diga quão } \\
\text { ideal se encontra a doçura da amostra }\end{array}$ & $\begin{array}{l}+3 \text { muito mais doce que o ideal } \\
+2 \\
1 \\
0 \text { ideal } \\
-1 \\
-2 \\
-3 \text { muito menos doce que o ideal }\end{array}$ \\
\hline $\begin{array}{l}6 \text { - Se você encontrasse este produto } \\
\text { à venda, indique através da escala ao } \\
\text { lado o grau de certeza que você } \\
\text { compraria ou não o produto }\end{array}$ & $\begin{array}{l}1 \text { certamente compraria } \\
2 \\
3 \text { talvez comprasse talvez não } \\
4 \\
5 \text { certamente não compraria }\end{array}$ \\
\hline
\end{tabular}

Figura 3. Ficha utilizada para o teste de aceitação.

de bebida láctea achocolatada avaliadas são apresentados na Tabela 3. O perfil de cada uma dessas marcas é mostrado graficamente na Figura 4, onde o valor médio atribuído pelos provadores a cada descritor é marcado no eixo correspondente. O centro da figura representa o ponto zero da escala utilizada na avaliação, enquanto a intensidade aumenta do centro para a periferia. Assim, o perfil sensorial se revela quando se faz a conexão dos pontos.

Os testes de ANOVA e Tukey (Tabela 3) mostraram que houve diferença significativa $(\mathrm{p}<0,05)$ entre pelo menos duas amostras, para todos os atributos avaliados. Observou-se que a bebida láctea da marca $\mathrm{A}$ distinguiu-se das demais por apresentar aroma e sabor de caramelo intensos, que podem estar relacionados com a maior doçura. A amostra B destacou-se pela alta intensidade da cor marrom e do brilho, como também pela aparência viscosa, maior textura e arenosidade. A amostra C, por sua vez, caracterizou-se por apresentar maior intensidade tanto no sabor/aroma de leite, como no aroma de chocolate. De forma geral, todos os produtos foram representados por 
Tabela 2. Desempenho dos provadores candidatos a equipe sensorial em relação a seu poder discriminatório $\left(\mathrm{P}_{\text {amostra }}\right)$ e repetibilidade $\left(\mathrm{P}_{\text {repetição }}\right)$. Valores desejáveis: $\mathrm{P}_{\text {amostra }}<0,30$ e Repetição $>0,05$.

\begin{tabular}{|c|c|c|c|c|c|c|c|}
\hline \multicolumn{2}{|r|}{ Descritor } & \multirow[t]{2}{*}{$\mathrm{P}$} & \multicolumn{5}{|c|}{ Provador } \\
\hline & & & 1 & 2 & 3 & 4 & 5 \\
\hline \multirow[t]{6}{*}{ Aparencia } & Cor marrom & $\mathrm{P}_{\mathrm{am}}$ & 0,0001 & 0,0001 & 0,0001 & 0,0009 & 0,0246 \\
\hline & & $\mathrm{P}_{\text {rep }}$ & 0,1898 & 0,8074 & 0,2524 & 0,7713 & 0,4987 \\
\hline & Brilho & $\mathrm{P}_{\mathrm{am}}$ & 0,1103 & 0,0354 & 0,0006 & 0,0029 & ${ }^{\star} 0,3038$ \\
\hline & & $\mathrm{P}_{\text {rep }}$ & 0,5646 & 0,5950 & 0,1349 & ${ }^{*} 0,0043$ & 0,6712 \\
\hline & Viscosidade & $\mathrm{P}_{\mathrm{am}}$ & 0,0001 & 0,0001 & 0,0004 & 0,0006 & 0,0933 \\
\hline & & $\mathrm{P}_{\text {rep }}$ & 0,3148 & 0,1127 & 0,3057 & ${ }^{\star} 0,0428$ & 0,8981 \\
\hline \multirow[t]{6}{*}{ Aroma } & Aroma chocolate & $\mathrm{P}_{\mathrm{am}}$ & 0,0001 & 0,1462 & 0,0020 & 0,0096 & 0,0179 \\
\hline & & $\mathrm{P}_{\text {rep }}$ & 0,8365 & 0,9907 & 0,5088 & 0,7255 & 0,1412 \\
\hline & Aroma leite & $\mathrm{P}_{\mathrm{am}}$ & 0,0014 & 0,0002 & 0,0006 & 0,0001 & ${ }^{*} 0,6682$ \\
\hline & & $\mathrm{P}_{\text {rep }}$ & 0,3893 & ${ }^{\star} 0,0325$ & 0,2914 & 0,0663 & 0,9482 \\
\hline & Aroma caramelo & $\mathrm{P}_{\mathrm{am}}$ & 0,0001 & 0,0130 & 0,0035 & 0,0001 & 0,0433 \\
\hline & & $\mathrm{P}_{\text {rep }}$ & 0,4582 & 0,5107 & 0,2837 & 0,2315 & 0,6366 \\
\hline \multirow[t]{8}{*}{ Sabor } & Sabor chocolate & $\mathrm{P}_{\mathrm{am}}$ & 0,0004 & ${ }^{*} 0,9155$ & 0,0016 & 0,0214 & 0,0006 \\
\hline & & $\mathrm{P}_{\text {rep }}$ & 0,8810 & 0,9178 & 0,3082 & 0,5955 & 0,2766 \\
\hline & Sabor caramelo & $\mathrm{P}_{\mathrm{am}}$ & 0,0001 & 0,0526 & 0,0005 & 0,0021 & ${ }^{*} 0,7680$ \\
\hline & & $P_{\text {rep }}$ & 0,8489 & 0,9094 & 0,9289 & 0,8243 & 0,7923 \\
\hline & Sabor leite & $\mathrm{P}_{\mathrm{am}}$ & 0,0060 & 0,0056 & 0,0008 & 0,0007 & 0,0344 \\
\hline & & $\mathrm{P}_{\text {rep }}$ & 0,0820 & 0,2667 & 0,2420 & 0,8482 & 0,6216 \\
\hline & Doçura & $\mathrm{P}_{\mathrm{am}}$ & 0,0001 & 0,0001 & 0,0001 & 0,0065 & ${ }^{\star} 0,8489$ \\
\hline & & $\mathrm{P}_{\text {rep }}$ & 0,0800 & 0,6208 & 0,8403 & 0,9537 & 0,9445 \\
\hline \multirow[t]{6}{*}{ Textura } & Corpo & $\mathrm{P}_{\mathrm{am}}$ & 0,0001 & 0,0001 & 0,0005 & 0,0001 & 0,0073 \\
\hline & & $\mathrm{P}_{\text {rep }}$ & 0,2891 & ${ }^{\star} 0,0236$ & 0,4242 & ${ }^{\star} 0,0257$ & 0,6498 \\
\hline & Arenosidade & $\mathrm{P}_{\mathrm{am}}$ & 0,0044 & 0,0002 & 0,0003 & 0,0001 & ${ }^{*} 0,9211$ \\
\hline & & $\mathrm{P}_{\text {rep }}$ & 0,5447 & 0,2024 & 0,6577 & 0,1091 & 0,9851 \\
\hline & ND & & 0 & 1 & 0 & 0 & 5 \\
\hline & NR & & 0 & 2 & 0 & 3 & 0 \\
\hline
\end{tabular}

* Valores não atendendo aos valores especificados para $\mathrm{P}_{\text {amostra }}$ e $\mathrm{P}_{\text {repetiçăo }} ; \mathrm{ND}=$ número de vezes que o provador não discriminou as amostras a $\mathrm{p}<0,30$; e $\mathrm{NR}=$ número de vezes que o provador não apresentou repetibilidade a $\mathrm{p}>0,05$.

Tabela 3. Médias dos valores atribuídos pelos provadores a cada descritor para as amostras de bebida láctea achocolatada.

\begin{tabular}{lccc}
\hline \multicolumn{1}{c}{ Descritores } & \multicolumn{3}{c}{ Amostras } \\
\cline { 2 - 4 } & $\mathrm{A}$ & $\mathrm{B}$ & $\mathrm{C}$ \\
\hline Cor marrom & $4,49^{\mathrm{b}}$ & $7,17^{\mathrm{a}}$ & $1,55^{\mathrm{c}}$ \\
Brilho & $6,52^{\mathrm{b}}$ & $7,07^{\mathrm{a}}$ & $6,28^{\mathrm{b}}$ \\
Viscosidade & $2,57^{\mathrm{b}}$ & $6,70^{\mathrm{a}}$ & $1,53^{\mathrm{c}}$ \\
Aroma chocolate & $2,03^{\mathrm{c}}$ & $3,52^{\mathrm{b}}$ & $6,75^{\mathrm{a}}$ \\
Aroma de leite & $2,06^{\mathrm{b}}$ & $1,68^{\mathrm{b}}$ & $6,40^{\mathrm{a}}$ \\
Aroma de caramelo & $7,55^{\mathrm{a}}$ & $3,12^{\mathrm{b}}$ & $2,16^{\mathrm{c}}$ \\
Sabor de chocolate & $2,33^{\mathrm{b}}$ & $4,80^{\mathrm{a}}$ & $5,81^{\mathrm{a}}$ \\
Sabor de caramelo & $7,66^{\mathrm{a}}$ & $3,84^{\mathrm{b}}$ & $2,00^{\mathrm{c}}$ \\
Sabor de leite & $2,38^{\mathrm{b}}$ & $1,63^{\mathrm{b}}$ & $6,33^{\mathrm{a}}$ \\
Doçura & $7,64^{\mathrm{a}}$ & $5,50^{\mathrm{b}}$ & $2,02^{\mathrm{c}}$ \\
Corpo & $2,28^{\mathrm{b}}$ & $6,83^{\mathrm{a}}$ & $1,43^{\mathrm{c}}$ \\
Arenosidade & $0,61^{\mathrm{c}}$ & $6,96^{\mathrm{a}}$ & $2,65^{\mathrm{b}}$ \\
\hline
\end{tabular}

a,b,c Médias na mesma linha acompanhadas de mesma letra não diferem entre si ao nível de $5 \%$ de significância.

diferentes atributos, que é uma relevante distinção entre eles em um espaço multidimensional.

Quando os resultados da Análise Descritiva Quantitativa da bebida láctea achocolatada foram submetidos à Análise

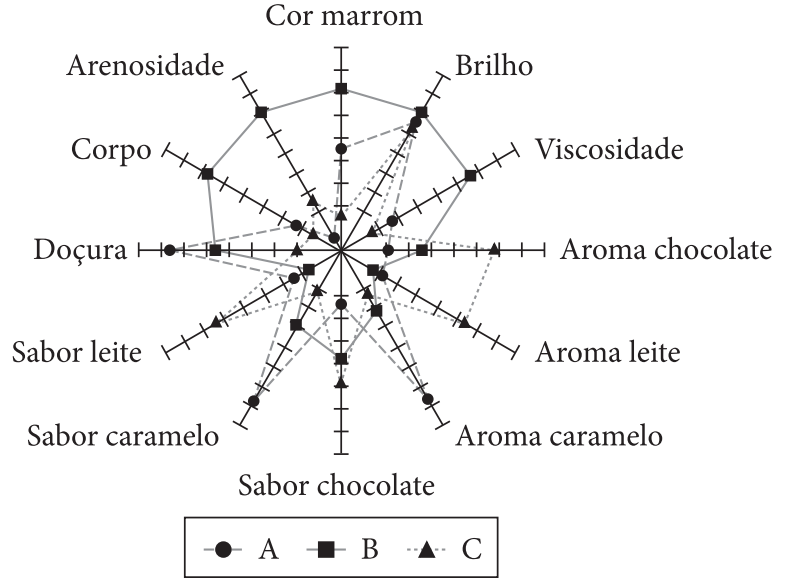

Figura 4. Perfil sensorial das amostras de bebida láctea achocolatada.

Multivariada de Componentes Principais (ACP), as características sensoriais de cada amostra foram evidenciadas, apresentando grande concordância com os resultados obtidos anteriormente. No entanto, para a análise de componentes principais, foram eliminados 4 atributos que constam no perfil sensorial, pois o gráfico deve conter um número de atributos 
menor que o número de amostras multiplicado pelas repetições $(3 \times 3)$. Isso explica o aparecimento de apenas oito vetores na Figura 5.

O método ACP é uma poderosa ferramenta estatística multivariada. Ele proporciona redução da dimensionalidade de um grupo de dados por formar combinações lineares das variáveis originais no estudo, as quais são chamadas componentes principais (PCs). Em geral essas combinações apresentam a maior contribuição para a variabilidade do estudo e são retidas nos dois PCs (KOZAC; SCAMAN, 2008). Muitas vezes, o ACP é utilizado para remover correlações entre variáveis antes do uso de outras análises, como análise discriminante ou regressão por mínimos quadrados parciais. Ressalte-se que esse método não é utilizado para previsão de uma variável dependente (COKER et al., 2005)

No gráfico da ACP, os descritores são representados como vetores, mencionados acima, os quais caracterizam as amostras que se localizam próximas a eles. As amostras são representadas por triângulos, sendo cada vértice uma repetição. Observa-se que houve boa discriminação entre as amostras, mesmo utilizando a forma monádica de apresentação. Resultados diferentes são reportados na ADQ de achocolatados, na apresentação monádica e simultânea das amostras. A segunda

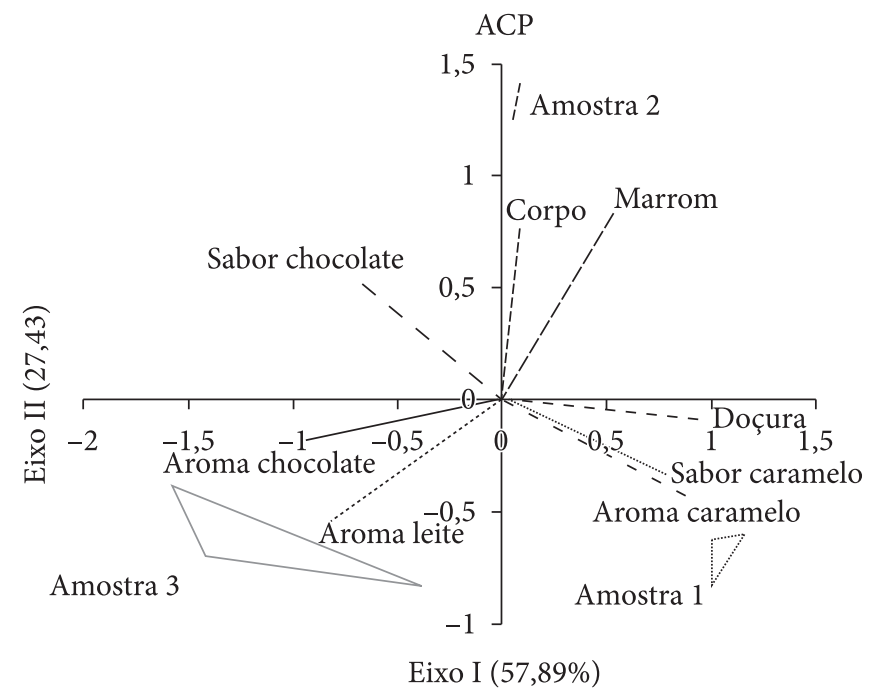

Figura 5. Projeção dos descritores sensoriais e amostras de bebida láctea achocolatada nos dois primeiros componentes principais. forma proporcionou uma melhor discriminação das amostras com relação aos descritores observados, sendo que $70 \%$ dos testes $\mathrm{F}$ das amostras obtidos foram mais elevados. De forma geral, observou-se uma discriminação melhor em 14 dos 20 atributos sensoriais, quando as amostras foram apresentadas simultaneamente (MAZZUCCHELLI; GUINARD, 1999).

A amostra de marca A apresentou maior intensidade de aroma e sabor de caramelo, enquanto que a amostra B caracterizou-se por maior intensidade de cor marrom e corpo. A amostra C foi caracterizada por apresentar elevado aroma de leite e de chocolate. Neste tipo de gráfico, quanto maior a decomposição do vetor nos eixos dos componentes, maior sua importância para caracterizar as diferenças entre as amostras. Dois componentes principais descreveram 85,32\% da variabilidade. Observou-se, neste caso, que para explicar os $57,89 \%$ da variabilidade entre as amostras, devido ao primeiro componente principal (CP1), foi importante a contribuição da maioria dos descritores analisados. A pouca variabilidade explicada pelo segundo CP foi devido principalmente ao corpo, ao aroma de leite e ao sabor de chocolate.

Na Tabela 4 são apresentados os coeficientes de correlação de Pearson entre os 8 descritores de bebida láctea achocolatada para as três marcas estudadas. Correlações esperadas entre os atributos foram reportadas. Observou-se alta correlação positiva entre o descritor sabor de caramelo com os descritores aroma de caramelo e doçura, a saber, 0,824 e 0,928; entre aroma de chocolate e aroma de leite $(0,914)$, vetores que estão muito próximos entre si na configuração bidimensional da ACP (Figura 5). Por outro lado, os descritores aroma e sabor de caramelo apresentaram alta correlação negativa com aroma e sabor de chocolate, sendo registrados valores de $-0,599$ e $-0,828$.

Thompson et al. (2004) utilizaram 4 PCs para descrever $72 \%$ da variabilidade presente em um estudo de bebidas lácteas achocolatadas comercializadas nos Estados Unidos. Intensidade de aroma e sabor de chocolate, aroma de caramelo, aroma cozido e intensidade de cor foram os principais descritores que caracterizam os dois primeiros PCs. Os autores relatam a existência de esperadas correlações entre os descritores, como entre aroma de chocolate e sabor de chocolate, sendo contudo um em um valor mais alto $(0,84)$ do que o registrado neste estudo (0,380). Isto pode estar relacionado a formulações das bebidas utilizadas e ao uso de aromas artificiais ou do próprio chocolate na formulação.

Tabela 4. Matriz de correlação entre os descritores de bebida láctea achocolatada para as três marcas avaliadas.

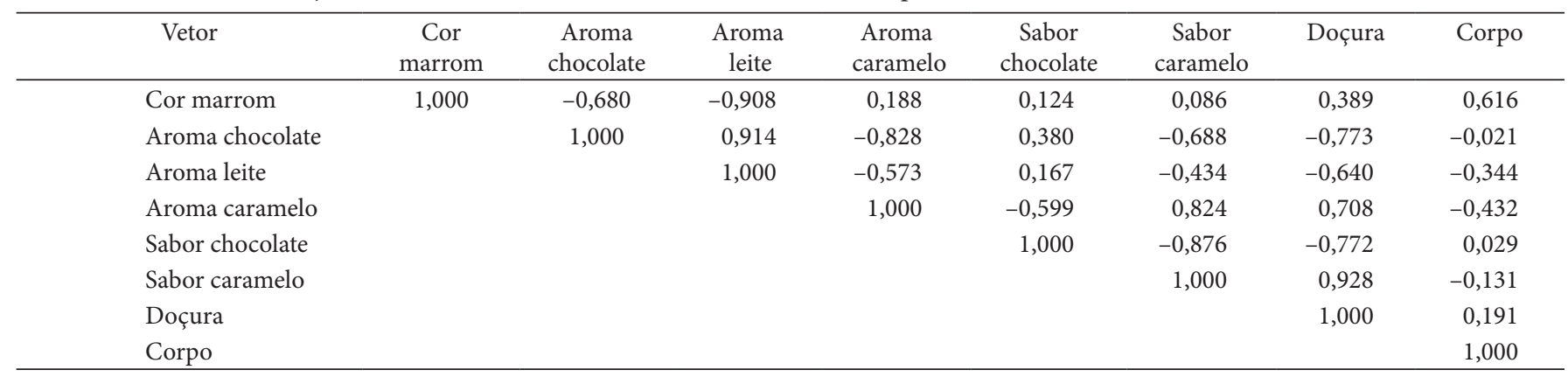




\subsection{Teste de aceitabilidade, intensidade de doçura e intenção de compra}

Através da análise dos resultados do teste de aceitação (Tabela 5), observou-se que as amostras de bebida láctea achocolatada foram igualmente aceitas pelos provadores quanto ao sabor e à forma global. Estes resultados mostram que a amostra líder de mercado (B) não obteve valores superiores para aceitação global e sabor diferente do que era esperado. Semelhantes resultados foram obtidos durante a aceitação de bebidas achocolatadas comerciais na Argentina, onde a marca conhecida e mais consumida pela população não foi a mais aceita (HOUGH et al., 1997).

Quando as amostras foram avaliadas quanto à intensidade de doçura (Figura 6), foi observado que a amostra B apresentou distribuição normal dos valores, sendo considerada com intensidade ideal de doçura, enquanto que a amostra A está levemente mais doce que o ideal, $\mathrm{e}$ a amostra $\mathrm{C}$ encontrou-se um

Distribuição de frequência da intensidade de doçura de bebida láctea achocolatada

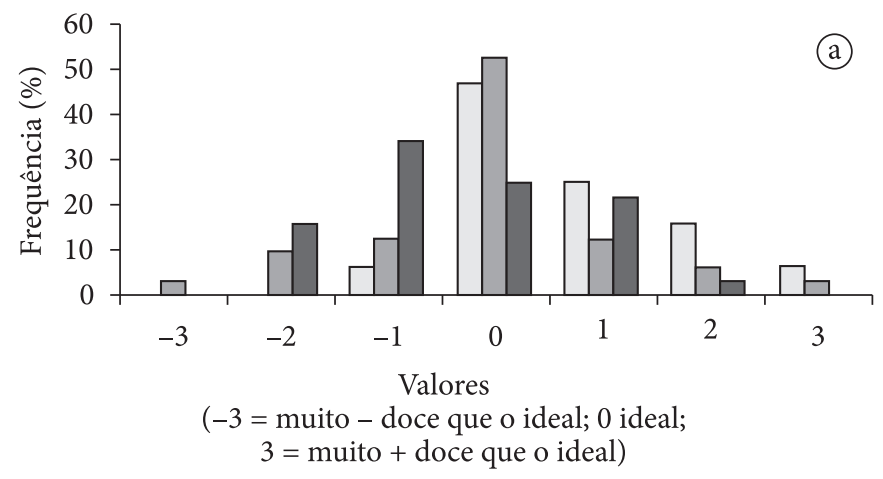

Distribuição de frequência da intenção de compra de bebida láctea achocolatada

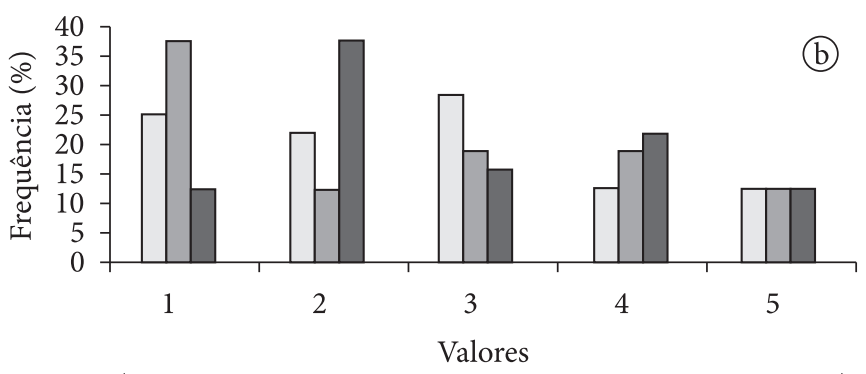

( 1 = certamente compraria; 5 = certamente não compraria $)$

$\square$ Amostra A $\square$ Amostra B $\square$ Amostra C

Figura 6. Histograma e freqüência dos valores atribuídos a intensidade de doçura e intenção de compra bebida láctea achocolatada.

Tabela 5. Médias dos valores atribuídos pelos provadores as três marcas de bebida achocolatadas, em uma escala hedônica de 9 pontos.

\begin{tabular}{lccc}
\hline \multicolumn{1}{c}{ Atributo } & Marca A & Marca B & Marca C \\
\hline Forma global $^{*}$ & $6,65^{\mathrm{a}}$ & $6,53^{\mathrm{a}}$ & $6,25^{\mathrm{a}}$ \\
Sabor $^{*}$ & $6,56^{\mathrm{a}}$ & $6,18^{\mathrm{a}}$ & $6,18^{\mathrm{a}}$ \\
\hline
\end{tabular}

${ }^{2}$ Médias na mesma linha acompanhadas de mesma letra não diferem entre si ao nível de $5 \%$ de significância; ${ }^{\star} 1$ = desgostei muitíssimo; e 9 = gostei muitíssimo pouco menos doce que o ideal. Estes resultados são coerentes com as diferenças significativas das médias encontradas para o descritor doçura na $\mathrm{ADQ}$, que obteve os valores 7,64; 5,50; $\mathrm{e}$ 2,02 para as amostras A, B e C, respectivamente.

Como observado na Figura 6, os valores encontrados para intenção de compra mostram uma tendência semelhante dos valores, sendo que um mesmo número de provadores atribuiu a nota de certamente não compraria, concluindo que houve pouca rejeição entre as amostras e que aproximadamente $50 \%$ dos provadores certamente ou provavelmente comprariam as 3 amostras avaliadas.

\section{Conclusões}

Um perfil sensorial para a bebida láctea achocolatada, consistindo em descritores de aparência, aroma, sabor e textura, foi estabelecido utilizando-se um conjunto de amostras comerciais. Dos doze descritores analisados, aroma e sabor de chocolate, aroma e sabor de caramelo, doçura e corpo foram considerados importantes na determinação da qualidade do produto, pois discriminaram claramente amostras de marcas distintas.

A bebida láctea achocolatada da marca A caracterizou-se por apresentar elevados valores de aroma e sabor de caramelo, enquanto que as características predominantes da marca B foram corpo e cor marrom muito intensos. A amostra C apresentou perfil oposto, caracterizada pela maior intensidade de aroma de leite e chocolate.

Todas as marcas analisadas foram bem aceitas pelos consumidores, em relação ao sabor e de uma forma geral, assim como a intenção compra. Com relação à doçura, os consumidores indicaram que a amostra B apresentava-se com doçura ideal e as amostras A e C estavam com doçura levemente mais e menos intensa, respectivamente.

\section{Referências bibliográficas}

ALMEIDA, K. E.; BONASSI, I. A.; ROÇA, R. O. Características físicas e químicas de bebidas lácteas fermentadas e preparadas com soro de queijo minas frescal. Ciência e Tecnologia de Alimentos, v. 21, n. 2, p. 187-192, 2001.

BRASIL. Ministério da Agricultura, Pecuária e Abastecimento. Instrução Normativa no ${ }^{\mathbf{1 6}}$, de 23 de agosto de 2005. Regulamento de Identidade e Qualidade de Bebidas Lácteas. Disponível em $<$ http:// www.agricultura.gov.br>. Acesso em: jun. 2006.

CASTRO, F. P. et al. Effect of oligofructose incorporation on the properties of fermented probiotic lactic beverages. International Journal of Dairy Technology, v. 62, n. 1, p. 68-74, 2009.

COKER, C. J. et al. Towards the classification of cheese variety and maturity on the basis of statistical analysis of proteolysis data - a review. International Dairy Journal, v. 15, n. 2, p. 631-643, 2005.

CRUZ, A. G. et al. Milk Drink Using Whey Butter Cheese (queijo manteiga) and Acerola Juice as a Potential Source of Vitamin C. Food and Bioprocess Technology, v. 2, n. 4, p. 368-373, 2009.

DAMASIO, M. H.; COSTELL, E. Análisis sensorial descriptivo: generación de descriptores y selección de catadores. Revista Agroquímica de Tecnología de Alimentos, v. 31, n. 2, p. 165-78, 1991. 
HOUGH, G. et al. Sensory optimization of a powdered chocolate milk formula. Food Quality and Preference, v. 8, n. 3, p. 213-221, 1997.

KEMP, S. E. Application of sensory evaluation in food research. International Journal of Food Science and Technology, v. 43, n. 9, p. 1507-1511, 2008.

KOFFI, E.; SHEWFELT, R.; WICKER, L. Storage stability and sensory analysis of UHT-processed whey-banana beverages. Journal of Food Quality, v. 28, n. 4, p. 386-401, 2005.

KOZAC, M.; SCAMAN, C. H. Unsupervised classification methods in food science: discussion and outlook. Journal of the Food Science and Agriculture, v. 88, n. 7, p. 1115-1117, 2008.

LIMA, S. M. C. G.; MADUREIRA, F. C. P.; PENNA, A. L. B. Bebidas lácteas - nutritivas e refrescantes. Milkbizz Tecnologia Temático, v. 1, n. 3, p. 4-11, 2002.

MAZZUCCHELLI, R.; GUINARD, J. X. Comparison of monadic and simultaneous sample presentations mode in a Descriptive analysis of milk chocolate. Journal of Sensory Studies, v. 14, n. 3 , p. 245-248, 1999.

MEHINAGIC, E. et al. Relationship between sensory analysis, penetrometry and visible NR spectroscopy of apples belonging to different cultivars. Food Quality and Preference, v. 14, n. 5, p. 473-484, 2003.

MEILGAARD, M.; CIVILLE, G. V.; CARR, B. T. Sensory evaluantion tecniques. Boca Raton: CRC Press, 1987.

MOSKOWITZ, H. R. Product testing and sensory evaluation of foods: marketing and R \& D approacher. Westport: Food and Nutrition Press, 1983. 605 p.

MURRAY, J. M.; DELAHUNTY, C. M.; BAXTER, I. A. Descriptive sensorial analysis: past, present and future. Food Research International, v. 34, n. 2, p. 461-471, 2001.
PIFARRE, A. M. et al. Acceptability and nutritional quality of a beverage based on orange juice and whey powder, preserved by heat or high-intensity pulsed electric fields (HIPEF). Archivos Latinoamericanos de Nutricion, v. 56, n. 4, p. 356-360, 2006.

RITJENS, S. Tendências mercadológicas dos iogurtes e bebidas lácteas. In: Leites fermentados e bebidas lácteas: Tecnologia e mercado. ITAL: Tecnolat, 1997.

SAS. User's procedures guide. Version 6. Cary: SAS Institute, Inc 1989. $2 \mathrm{v}$

SIMPSON, S. J.; PIGGOTT, J. R.; WILLIAMS, S. A. R. Sensorial Analysis. International Journal of Food Science and Technology, v. 33, n. 1, p. 7-18, 1998.

SIVIERI, K.; OLIVEIRA, M. N. Shelf-life evaluation of latic beverages added with fat with mimetics (Litesse and Dairy-Lo). Ciência e Tecnologia de Alimentos, v. 22, n. 1, p. 24-31, 2002.

STONE, H.; SIDEL, J. L. Descriptive analysis. In: STONE, H.; SIDEL, J. L. Sensory Evaluation Practices. London: Academic Press, 1985. p. 202-226.

THOMPSON, J. L. et al. Preference mapping of commercial chocolates milks. Journal of Food Science, v. 69, n. 9, p. S-406-S413, 2004.

TRANJAN, B. C. et al. Development of Whey Goat Beverages. International Journal of Dairy Technology, v. 62, n. 3, p. 438-443, 2009.

YANES, M.; DURÁN, L.; COSTELL, E. Rheological and optical properties of commercial chocolate milk beverages. Journal of Food Engineering, v. 51, n. 2, p. 229-234, 2002.

ZOELLNER, S. S. et al. Whey beverage containg açaí pulp as food Carrier for probiotic bactéria. Australian Journal of Dairy Technology, v. 64, n. 2, p. 165-169, 2009. 